\title{
Multi-scale Imaging of Al-7at.\%Cu Eutectics using Micro- and Nano-scale X-ray Computed Tomography
}

\author{
Brian M. Patterson ${ }^{1}$, Kevin C. Henderson ${ }^{1}$, Paul J. Gibbs ${ }^{1}$, Seth Imhoff $^{1}$, and Amy J. Clarke \\ ${ }^{1}$ Los Alamos National Laboratory, Materials Science and Technology Division, Los Alamos, USA.
}

Multi-scale X-ray computed tomography has the ability in the laboratory to answer a variety of questions related to the structure of materials, as well as the resultant differentiation of materials during solidification. Laboratory based 'micro-scale' systems (i.e., imaging with fields of view from 1 to 10's of $\mathrm{mm}$ ) can produce $3 \mathrm{D}$, non-destructive images with $\sim 1-\mu \mathrm{m}$ resolution. This scale is useful for mesoscale structure and metallic primary solidification dendritic structures and can be used to examine structural morphology and changes in morphology as a result processing both quantitatively and nondestructively and in three dimensions [1-3]. 'Nano-scale' CT has the ability to measure $\sim 100 \mu \mathrm{m}$ field of view down to $\sim 10$ 's of $\mu \mathrm{m}$ field of view, with resolution of $\sim 50 \mathrm{~nm}$. These systems can be used to measure micro-scale features and solidification eutectics.

Aluminum-copper eutectics are ideal systems for the study of solidification due to their high X-ray contrast and well differentiated structures; an added bonus is that the resultant microstructure has features on two length scales. A typical 'micro-scale' CT is shown in Figure 1, highlighting the separation of the $\mathrm{Al}$ and $\mathrm{Cu}$-containing regions. The primary dendritic structure, with ligaments 20-100 $\mu \mathrm{m}$ in thickness, lends itself very well to 'micro-scale' $\mathrm{CT}$. Within the $\mathrm{Cu}$-containing regions, eutectic structures with $\sim 2 \mu \mathrm{m}$ thick lamella are present and are ideal for imaging with 'nano-scale' CT. Three $100 \mu \mathrm{m}$ thick foils of an Al-7at.\%Cu alloy were melted and cooled in a boron nitride crucible at various cooling rates. The cooling rates were $0.44,0.67$, and $1.33{ }^{\circ} \mathrm{C} / \mathrm{s}$, in order to develop distinct microstructures. Micro-scale CT was used to 'pre-screen' samples and locate areas of interest, as well as aid in determining the 'global' structure of the material. Figure 2 shows a series of radiographs of a foil, imaged using micro-CT and stitching a series of radiographs together (Figure 2a). A 60 $\mu$ m diameter post was then machined and is shown relative to the original radiograph. Finally, the samples were then radiographed using a nano-scale CT (Figure 2c).

Results indicate that there are differences in the secondary dendritic arm spacing's seen in the radiographs of the three foils at the 'micro-scale'. Reconstructed slices (Figure 3(left)) show the $\sim 1-\mu \mathrm{m}$ lamella present in the $\mathrm{Cu}$-containing eutectic. Overlaying the images from the two length scales (Figure 3(middle)) and finally measuring the lamella thicknesses (Figure 3 (right)) gives an indication of the effect of cooling rate upon solidification. The thickness of the lamella decreases linearly with an increasing cooling rate[4].

\section{References:}

[1] B Patterson, E Cerreta, D Dennis-Koller, C Bronkhorst, B Hansen (2010) Microscopy and Microanalysis 16: 726.

[2] BM Patterson, JP Escobedo-Diaz, D Dennis-Koller, EK Cerreta (2012) Microscopy and Microanalysis 18: 390.

[3] BM Patterson, K Henderson, Z Smith (2012) Journal of Materials Science 48: 1986. Doi:10.1007/s10853-012-6965-2 
[4] We gratefully acknowledge support from AJC's Early Career award from the U.S. DOE, Division of Materials Sciences and Engineering.

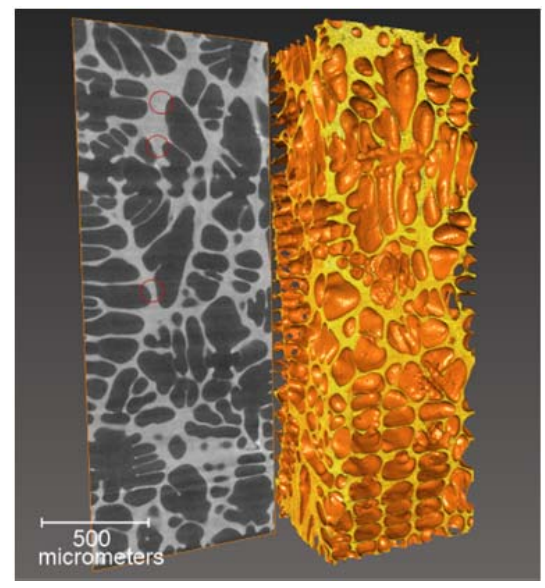

Figure 1: Micro-scale X-ray $\mathrm{CT}$ of an $\mathrm{Al}-7$ at. $\% \mathrm{Cu}$ sample. The $\mathrm{Cu}$-containing region is lighter in the reconstructed slice and rendered in $3 \mathrm{D}$ on the right. Eutectic structure is beginning to be resolved and a couple of features are seen in this $1.21 \mu \mathrm{m}$ isotropic voxel sized image (red circles).

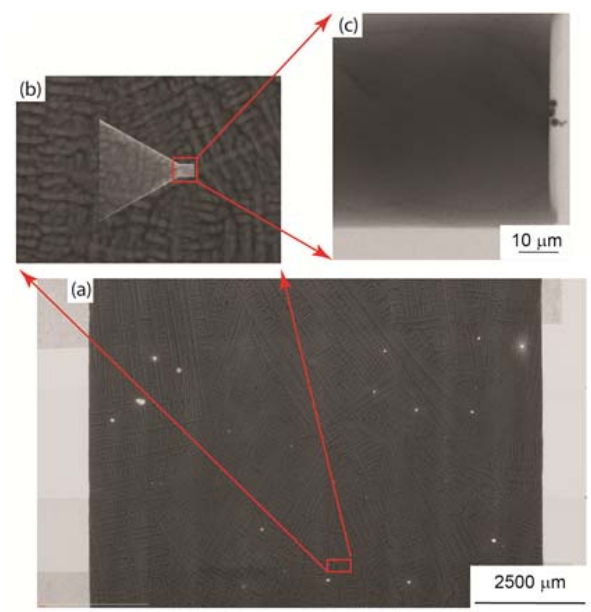

Figure 2: Successively higher resolution radiographs of a thin film of Al-7at.\%Cu. (a) has voxel size of $2.48 \mu \mathrm{m}$. (b) is an overlay of the radiography with the piece after it has been machined into a $60 \mu \mathrm{m}$ post. (c) shows the same post radiographed with a 'nano-scale' tomography instrument.
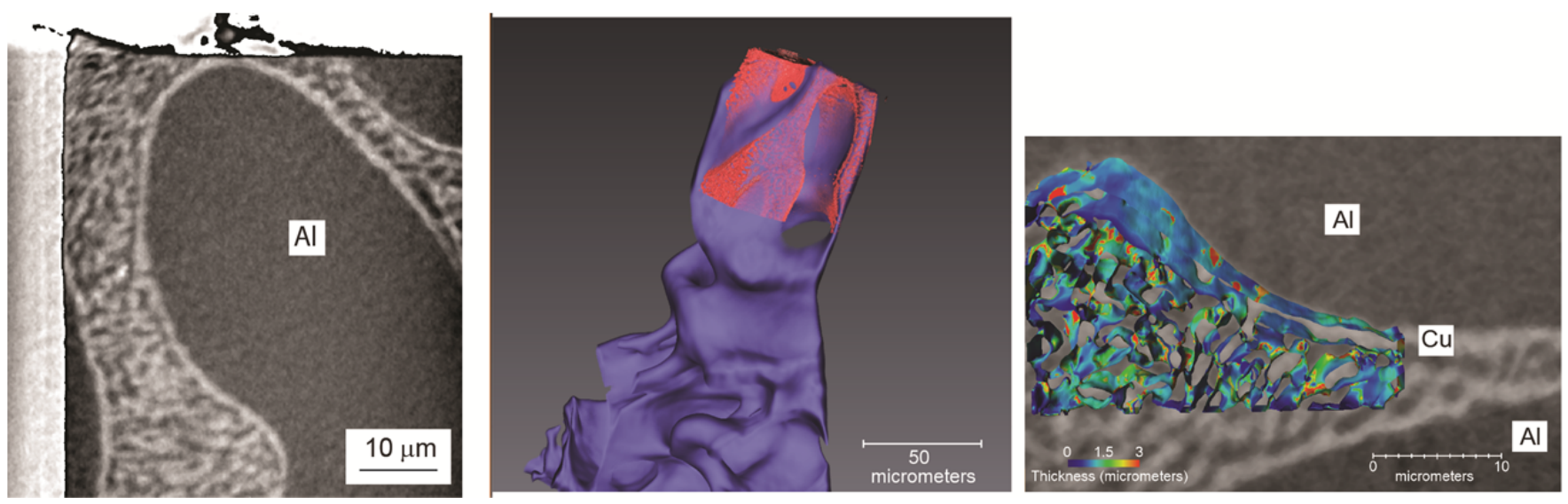

Figure 3: Reconstructed slice (left), overlay of the 'nano-scale' (65- $\mu \mathrm{m}$ voxel) and the 'micro-scale' image of the Al-7at.\%Cu eutectic (middle), and a measurement of the eutectic lamella thickness (right). 